\title{
DFT Study on the Oxidative Addition of 4-Substituted Iodobenzenes on $\operatorname{Pd}(0)$-Phosphine Complexes
}

\author{
Tímea R. Kégl, ${ }^{1,2,3}$ László Kollár, ${ }^{1,2,3}$ and Tamás Kégl ${ }^{1,2,3}$ \\ ${ }^{1}$ Department of Inorganic Chemistry, University of Pécs, Ifjúság Útja 6, Pécs 7624, Hungary \\ ${ }^{2} J a ́ n o s$ Szentágothai Research Centre, Ifjúság Útja 20, Pécs 7624, Hungary \\ ${ }^{3}$ MTA-TKI Research Group for Selective Chemical Syntheses, Pécs 7624, Hungary
}

Correspondence should be addressed to Tamás Kégl; tkegl@gamma.ttk.pte.hu

Received 31 July 2015; Accepted 10 September 2015

Academic Editor: Dennis Salahub

Copyright (C) 2015 Tímea R. Kégl et al. This is an open access article distributed under the Creative Commons Attribution License, which permits unrestricted use, distribution, and reproduction in any medium, provided the original work is properly cited.

\begin{abstract}
The oxidative addition of 4-substituted iodobenzenes on $\mathrm{Pd}(0)-\mathrm{PMe}_{3}$ complexes has been studied at the BP86 level of theory including dispersion correction and solvation effect, with tetrahydrofuran as solvent. The bisphosphine pathway was found to be barrierless, whereas the monophosphine route is hampered by the high dissociation energy of trimethylphosphine. The reaction free energy of this step shows linear correlation with the Hammett constants of the para substituents with the most electron withdrawing groups being the most exergonic.
\end{abstract}

The present scientific contribution is dedicated to the 650th anniversary of the foundation of the University of Pécs, Hungary

\section{Introduction}

Palladium-catalyzed cross-coupling reactions are the most prominent examples in the family of carbon-carbon bondforming reactions, which have gained enormous popularity in the last few decades $[1,2]$ and are found in all areas of chemistry, from polymers to pharmaceuticals and agrochemicals [3]. The Suzuki-Miyaura reaction is one of the most frequently used methods to form $\mathrm{C}-\mathrm{C}$ bond in a catalytic way [4], but the number of applications employing Sonogashira coupling is drastically increased in the recent years as well [5]. Other Pd-catalyzed cross-coupling reactions, such as Stille [6, 7], Tamao et al. [8], and Baba and Negishi [9] couplings, have their important role in organic synthesis as well.

In general, the initial step in the catalytic cycle is the oxidative addition of an aryl halide to palladium $(0)$ with cleavage of the C-X bond [10], which is followed by transmetallation and reductive elimination. The oxidative addition step has been the subject of several experimental [11-14] and computational [15-22] studies. Investigations regarding the entire catalytic cycle of Heck $[23,24]$ and Suzuki $[25,26]$ have been also reported.
In general, the barriers of oxidative addition of aryl halides increase in the order of $\mathrm{ArI}<\mathrm{ArBr}<\mathrm{ArCl}$ consistent with the reactivity order reported by Fitton and Rick [27]. Aryl halides with electron withdrawing groups give smaller barriers than those having electron donating groups [10]. As a catalytically active complex, $\operatorname{Pd}(0) \mathrm{L}_{2}$ is usually considered as reference point due to its relative stability. The $\mathrm{Pd}(0) \mathrm{L}_{2}$ complex then reacts with the aryl halide in a concerted manner affording the complex $\mathrm{Pd}(\mathrm{II}) \mathrm{X}_{2} \mathrm{~L}_{2}(\mathrm{X}=$ halide). Depending on the basicity, and the bulk of the phosphine, PdL complexes, formed after the dissociation of one phosphine ligand, should be considered as viable alternatives for active catalysts in some specific cases $[18,19]$. The bisphosphine and monophosphine pathways are illustrated in Scheme 1.

The goal of this study is to explore how the substituents in para position influence the energetics of the oxidative addition of iodobenzenes on $\mathrm{Pd}(0)-\mathrm{PMe}_{3}$ complexes taking into consideration both the bisphosphine and monophosphine pathways. Our objective is also to get some insight about the electronic structure of $\mathrm{Pd}-\mathrm{PhI}$ adducts which occur in both pathways. 


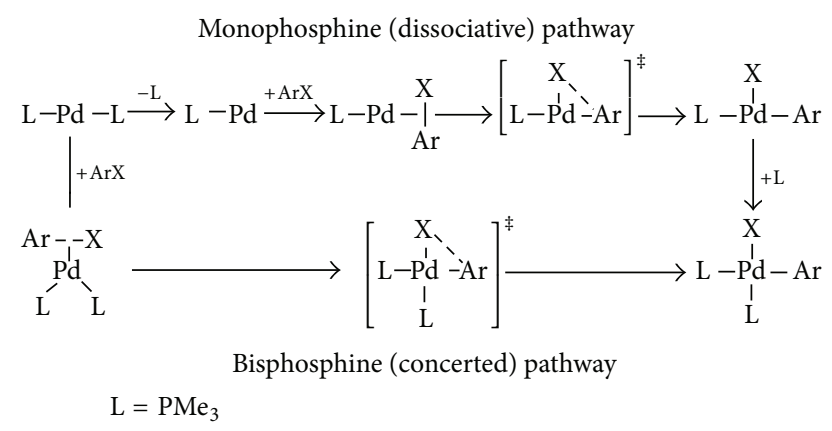

Scheme 1: Comparison of the bisphosphine and monophosphine pathways.

\section{Computational Details}

All structures were fully optimized at the DFT/BP86 level of theory $[28,29]$ with ultrafine grids, assuming THF as solvent, employing the Gaussian 09 suite of programs [30]. The dispersion correction developed by Grimme et al. has been employed with Becke-Johnson damping and denoted by BP86-D3 [31]. For palladium and iodine the def2-TZVP whereas for the other atoms the def2-SVP basis sets were used [32]. Local minima were identified by the absence of the negative eigenvalues in the vibrational frequency analyses, whereas the Hessian matrix of transition states has only one negative eigenvalue. Gibbs free energies were calculated at $298.15 \mathrm{~K}$. Intrinsic reaction coordinate (IRC) analyses [33] were carried out at the same level as the geometry optimizations in order to make sure that the corresponding local minima and transition states are smoothly connected to each other.

The effect of the solvent was taken into account utilizing the SMD solvation model [34], introduced by Marenich et al., with the dielectric constant of $\epsilon=7.4257$ for tetrahydrofuran. The SMD method is based on the charge density of a solute molecule interacting with the solvent represented as continuum. The full solute electron density is employed without the definition of partial atomic charges; therefore, " $D$ " stands for "density" in the abbreviation of the model. As in the previous SMx solvation models $(x=1-8)$ developed by the Minnesota group the solvent is represented as a dielectric medium with surface tension at the solute-solvent boundary.

For the QTAIM (Quantum Theory of Atoms In Molecules) calculations the AIMAll software package was utilized [35].

\section{Results and Discussion}

It is known that increasing the phosphine basicity is advantageous to the activity of the catalytic system. It was also shown that sterically more demanding phosphines tend to turn the monoligated pathway as the preferred one. For this study the less bulky, yet strongly basic trimethylphosphine has been selected. For catalytically active complexes both $\mathrm{Pd}\left(\mathrm{PMe}_{3}\right)_{2}$ (1) and $\mathrm{Pd}\left(\mathrm{PMe}_{3}\right)$ (2) have been taken into consideration in order to decide whether the monoligated or the bisligated pathways are dominating for the various

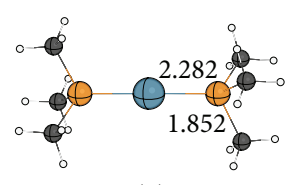

(1)

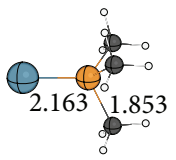

(2)

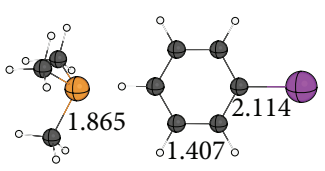

(3a)
Figure 1: Computed structures of the initial compounds for the oxidative addition step: $\mathrm{Pd}\left(\mathrm{PMe}_{3}\right)_{2}$ (1), $\mathrm{Pd}\left(\mathrm{PMe}_{3}\right)(2), \mathrm{PMe}_{3}$, and iodobenzene (3a). Bond distances are given in $\AA$.

substituted iodobenzenes at the BP86/SMD level of theory including dispersion correction.

The dissociation of one $\mathrm{PMe}_{3}$ from complex (1) undergoes with a free energy of $31.2 \mathrm{kcal} / \mathrm{mol}$, which is notably higher than that obtained by Lam and coworkers $(20.5 \mathrm{kcal} / \mathrm{mol})$ at B3LYP level with double- $\zeta$ basis set, without solvation corrections [19]. As expected, the remaining $\mathrm{PMe}_{3}$ is bound stronger to $\mathrm{Pd}$, which is reflected in the shorter Pd-P bond distance $(2.163 \AA$ ) as opposed to that in complex $1(2.282 \AA)$ (Figure 1).

In order to unravel the strong dependence of the dissociation free energy $\left(\Delta G_{\text {diss }}\right)$ of trimethylphosphine from 1 the reaction free energy for the $\mathbf{1} \rightarrow \mathbf{2}+\mathrm{PMe}_{3}$ process has been recomputed with the BP86 functional investigating the effect of solvation and dispersion corrections. Surprisingly, the neglect of solvent effects resulted in a $\Delta G_{\text {diss }}$ being only slightly higher than the original value $(32.2 \mathrm{kcal} / \mathrm{mol})$, whereas at the BP86/SMD level (without D3 correction) $24.7 \mathrm{kcal} / \mathrm{mol}$ has been obtained, being fairly close to $\Delta G_{\text {diss }}=$ $20.5 \mathrm{kcal} / \mathrm{mol}$ achieved with the B3LYP functional [19].

For the pathway involving complex (1) iodobenzene coordinates with the palladium center in a $\eta^{2}$-like manner (Figure 2) resulting in the adduct 4a. The coordination causes a slight increase in Pd-P bond length, as well as in the distance of the coordinating carbon atoms going from $1.407 \AA$ to $1.462 \AA$. The coordination is exergonic by $-7.3 \mathrm{kcal} / \mathrm{mol}$.

The oxidative addition step itself is very facile. The free energy difference between $\mathbf{4 a}$ and transition state $\mathbf{5 T S a}$ is $6.7 \mathrm{kcal} / \mathrm{mol}$, meaning that the overall activation free energy is $-0.6 \mathrm{kcal} / \mathrm{mol}$; thus, it is below the sum of the free energies of the separated reactants. As a result of this process a somewhat distorted square planar $\mathrm{Pd}(\mathrm{II})$-iodo-phenyl complex is formed (6a) where the phenyl group is perpendicular to the plane spanned by the phosphines and the iodo ligand. The formation of $\mathbf{6 a}$ is exergonic by $-36.7 \mathrm{kcal} / \mathrm{mol}$ with respect to the summed free energies of $\mathbf{1}$ and $\mathbf{3 a}$.

The dissociative pathway starts with the dissociation of one $\mathrm{PMe}_{3}$ with a bond dissociation free energy of $31.2 \mathrm{kcal} / \mathrm{mol}$ (vide supra) giving rise to the monoligated complex $\mathrm{Pd}\left(\mathrm{PMe}_{3}\right)$ (2). The coordination of iodobenzene (3a) is notably more exergonic $(-17.8 \mathrm{kcal} / \mathrm{mol})$ in comparison to that of the more saturated complex (1). The coordination type is, however, more like $\eta^{1}-\mathrm{C}$, compared to $\eta^{2}$ (C,C) according to the significant difference in bond lengths between palladium and the coordinating carbon atoms. The oxidative addition step then proceeds via transition state 8TSa with a barrier of $0.8 \mathrm{kcal}$. The formation of the T-shaped $\mathrm{Pd}(\mathrm{II})$ complex $9 \mathbf{a}$ is endergonic by $27.3 \mathrm{kcal} / \mathrm{mol}$. The closing step of the dissociative oxidative addition pathway is the 


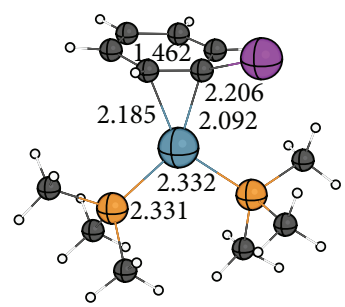

$(4 a)$

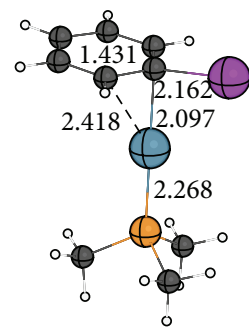

(7a)

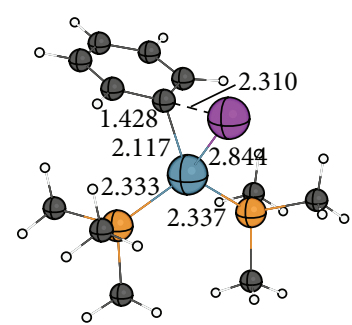

(5TSa)

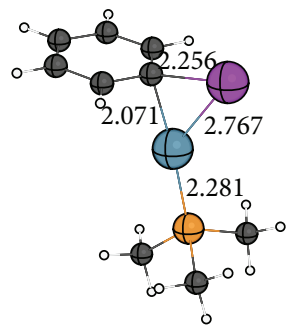

(8TSa)

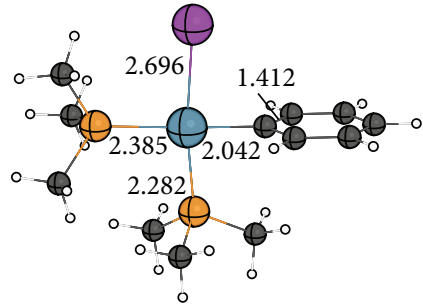

(6a)

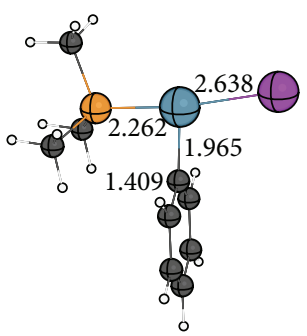

(9a)

Figure 2: Computed structures of the intermediates and transition states associated with the oxidative addition step. Bond distances are given in $\AA$.

coordination of $\mathrm{PMe}_{3}$ to the vacant site on Pd resulting in 6a. The free energy associated with this process is $-13.1 \mathrm{kcal} / \mathrm{mol}$, meaning that trimethylphosphine is much less weakly bound in $\mathbf{6} \mathbf{a}$ than in $\mathbf{1}$.

The concerted and the dissociative pathways are compared in Figure 3. A clear preference for the former one is predicted at the BP86-D3 level of theory with virtually no barrier due to the relatively high stability of the transition state 5TSa.

In order to get more insight into the electronic structure of iodobenzene adducts and the oxidative addition transition state, QTAIM calculations [36] have been performed and the Laplacians of the electron densities are depicted in Figure 4. The visual inspection of the Laplacian distribution of $\mathbf{4 a}$ shows a close resemblance to that of olefin complexes, for instance, in $\mathrm{W}(\mathrm{CO})_{5}\left(\mathrm{C}_{2} \mathrm{H}_{4}\right)$ [37], where the distortion in electron density distribution also emphasizes the presence of a donor interaction. The existence of bond paths between $\mathrm{Pd}$ and two carbons in iodobenzene suggests that the coordination mode is indeed $\eta^{2}-(C, C)$. On the other hand, the corresponding carbon atoms in 7 a show difference not only in the distance to $\mathrm{Pd}$, but also in the lack of the second Pd-C bond path, and much less distortion in the density distribution of the second carbon. Hence, the coordination mode in the more unsaturated Pd-iodobenzene complex is undoubtedly of $\eta^{1}$-C type.

The Laplacian distribution of transition state 5TSa reveals the presence of a three-center Pd-C-I bond with bond paths between these three atoms. The electron density distribution around the carbon atom shows significant distortion towards the iodine, which may be responsible for the high relative stability of 5TSa in terms of free energy.

The substituent effect for the oxidative addition of 4substituted iodobenzenes was investigated with some representative functional groups spanning the range of Hammett

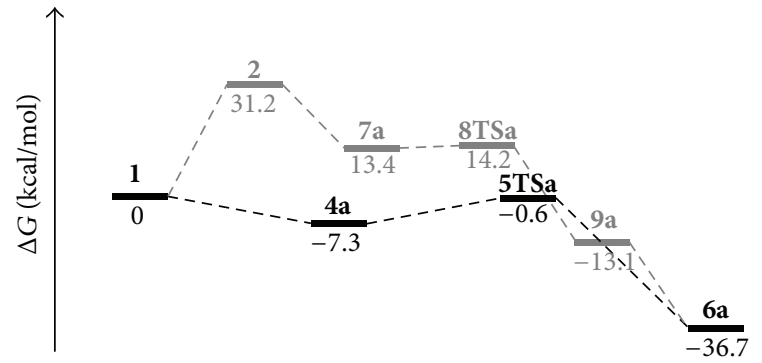

FIGURE 3: Gibbs free energy diagram of the oxidative addition for the concerted (black) and the dissociative (grey) pathways.

para constants between -0.83 and 0.78 . The free energies of the iodobenzene coordination, the oxidative addition, and the activation free energies were collected in Table 1 for the bisphosphine pathways and in Table 2 for the monophosphine pathways.

In terms of the bond free energy between the iodobenzenes and the palladium containing fragment the substituents show a quite strong dependence on $\sigma_{p}$. For the electron donor groups, such as OMe, and Me the coordination strength is rather weak. Moreover, the iodobenzene coordination and dissociation are almost in equilibrium in the case of the $\mathrm{NMe}_{2}$ substituent. On the other hand, for the strongly electron withdrawing groups $\mathrm{CN}$ and $\mathrm{NO}_{2}$ the free energy of coordination exceeds $-10 \mathrm{kcal} / \mathrm{mol}$.

Interestingly, the free energy difference between the adducts (4) and the transition states (5TS) shows notable difference only for the most electron donating substituents. For $\mathrm{NMe}_{2}$, and $\mathrm{OMe}$, this difference is only 1.7 and $2.0 \mathrm{kcal} / \mathrm{mol}$, respectively. On the other hand, it is $5.0 \mathrm{kcal} / \mathrm{mol}$ for the methyl group and $6.2 \mathrm{kcal} / \mathrm{mol}$ for the nitro group, and difference in free energies falls in this range for all the 
TABLE 1: Computed activation free energies and reaction free energies (in $\mathrm{kcal} / \mathrm{mol}$ ) for the oxidative addition of 4 -substituted iodobenzenes following the bisphosphine pathway.

\begin{tabular}{|c|c|c|c|c|c|}
\hline Substituent & $\sigma_{p}$ & Pathway & $\Delta G_{1}$ & $\Delta G^{\ddagger}$ & $\Delta G_{2}$ \\
\hline $\mathrm{NMe}_{2}$ & -0.83 & $1+3 b \rightarrow 4 b \rightarrow 5 \mathrm{TSb} \rightarrow 6 \mathrm{~b}$ & -0.1 & 1.6 & -31.7 \\
\hline $\mathrm{OMe}$ & -0.37 & $1+3 c \rightarrow 4 c \rightarrow 5 T S c \rightarrow 6 c$ & -2.7 & -0.7 & -32.9 \\
\hline $\mathrm{Me}$ & -0.17 & $1+3 d \rightarrow 4 d \rightarrow 5 T S d \rightarrow 6 d$ & -4.0 & 1.0 & -35.5 \\
\hline $\mathrm{H}$ & 0 & $1+3 a \rightarrow 4 a \rightarrow 5 T S a \rightarrow 6 a$ & -7.3 & -0.6 & -36.7 \\
\hline $\mathrm{Cl}$ & 0.23 & $1+3 \mathrm{e} \rightarrow 4 \mathrm{e} \rightarrow 5 \mathrm{TSe} \rightarrow 6 \mathrm{e}$ & -6.0 & -0.1 & -37.7 \\
\hline $\mathrm{CF}_{3}$ & 0.54 & $1+3 f \rightarrow 4 f \rightarrow 5 T S f \rightarrow 6 f$ & -8.3 & -1.7 & -38.1 \\
\hline $\mathrm{CN}$ & 0.66 & $1+3 \mathrm{~g} \rightarrow 4 \mathrm{~g} \rightarrow 5 \mathrm{TSg} \rightarrow 6 \mathrm{~g}$ & -11.7 & -3.8 & -39.5 \\
\hline $\mathrm{NO}_{2}$ & 0.78 & $1+3 h \rightarrow 4 h \rightarrow 5$ TSh $\rightarrow 6 h$ & -11.4 & -5.2 & -39.9 \\
\hline
\end{tabular}

${ }^{\ddagger}$ The gibbs free energy of activation.

TABLE 2: Computed activation free energies and reaction free energies (in kcal/mol) for the oxidative addition of 4-substituted iodobenzenes following the dissociated pathway. The opening step that is the dissociation of one trimethylphosphine needs a free energy of $31.2 \mathrm{kcal} / \mathrm{mol}$. Free energy values are relative to the sum of those of $\mathrm{Pd}\left(\mathrm{PMe}_{3}\right)_{2}$ and the corresponding iodobenzenes.

\begin{tabular}{|c|c|c|c|c|c|}
\hline Substituent & $\sigma_{p}$ & Pathway & $\Delta G_{1}$ & $\Delta G^{\ddagger}$ & $\Delta G_{2}$ \\
\hline $\mathrm{NMe}_{2}$ & -0.83 & $\left(1-\mathrm{PMe}_{3} \rightarrow\right) 2+3 \mathrm{~b} \rightarrow 7 \mathrm{~b} \rightarrow 8 \mathrm{TSb} \rightarrow 9 \mathrm{~b}$ & 3.5 & 14.6 & -10.9 \\
\hline $\mathrm{OMe}$ & -0.37 & $\left(1-\mathrm{PMe}_{3} \rightarrow\right) 2+3 \mathrm{c} \rightarrow 7 \mathrm{c} \rightarrow 8 \mathrm{TSc} \rightarrow 9 \mathrm{c}$ & 3.0 & 15.8 & -11.3 \\
\hline $\mathrm{Me}$ & -0.17 & $\left(1-\mathrm{PMe}_{3} \rightarrow\right) 2+3 \mathrm{~d} \rightarrow 7 \mathrm{~d} \rightarrow \mathbf{8 T S d} \rightarrow 9 \mathrm{~d}$ & 5.8 & 15.5 & -11.2 \\
\hline $\mathrm{H}$ & 0 & $\left(1-\mathrm{PMe}_{3} \rightarrow\right) 2+3 \mathrm{a} \rightarrow 7 \mathrm{a} \rightarrow 8 \mathrm{TSa} \rightarrow 6 \mathrm{a}$ & 13.4 & 14.2 & -13.1 \\
\hline $\mathrm{Cl}$ & 0.23 & $\left(1-\mathrm{PMe}_{3} \rightarrow\right) 2+3 \mathrm{e} \rightarrow 7 \mathrm{e} \rightarrow 8 \mathrm{TSe} \rightarrow 9 \mathrm{e}$ & 11.8 & 13.0 & -12.0 \\
\hline $\mathrm{CF}_{3}$ & 0.54 & $\left(1-\mathrm{PMe}_{3} \rightarrow\right) 2+3 \mathrm{f} \rightarrow 7 \mathrm{f} \rightarrow \mathbf{8 T S f} \rightarrow$ 9f & 11.9 & 12.6 & -10.5 \\
\hline $\mathrm{CN}$ & 0.66 & $\left(1-\mathrm{PMe}_{3} \rightarrow\right) 2+3 \mathrm{~g} \rightarrow 7 \mathrm{~g} \rightarrow 8 \mathrm{TSg} \rightarrow 9 \mathrm{~g}$ & 11.0 & 11.7 & -13.0 \\
\hline $\mathrm{NO}_{2}$ & 0.78 & $\left(1-\mathrm{PMe}_{3} \rightarrow\right) 2+3 \mathrm{~h} \rightarrow 7 \mathrm{~h} \rightarrow 8 \mathrm{TSh} \rightarrow 9 \mathrm{~h}$ & 10.6 & 12.2 & -13.6 \\
\hline
\end{tabular}

${ }^{\ddagger}$ The gibbs free energy of activation.

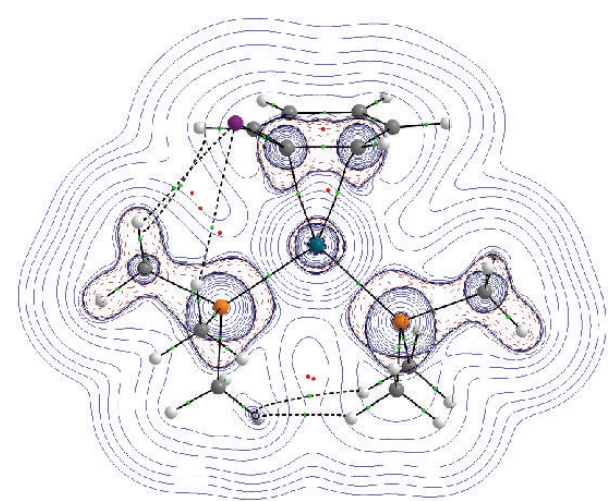

(4a)

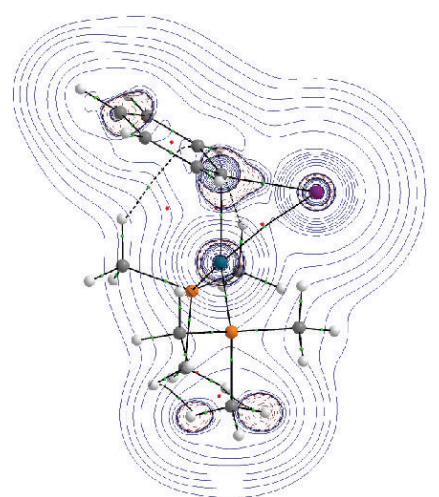

$(5 \mathrm{TSa})$

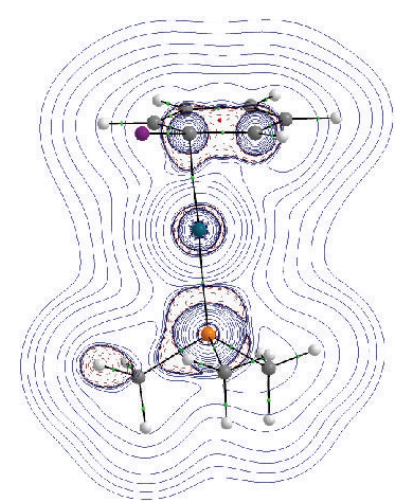

(7a)

Figure 4: Laplacian $\left(\nabla^{2} \rho(\mathbf{r})\right)$ of the electron density of iodobenzene adducts (4a) and (7a) as well as that of transition state (5TSa). The charge concentration regions $\left(\nabla^{2} \rho(\mathbf{r})<0\right)$ are designated with dashed lines.

other substituents. It is important to emphasize, however, that the oxidative addition is very facile in the case of the bisphosphine pathway, regardless of the substituents on iodobenzene, and can be considered as barrierless. This is in contrast with the experimental [13] and theoretical [20] findings with basic, but bulky, phosphines, where the free energy barrier for the oxidative addition of $\mathrm{PhI}$ was found as
24.6 and $28.4 \mathrm{kcal} / \mathrm{mol}$, respectively, emphasizing the key role of the ligand bulk upon the reaction mechanism.

The reaction free energies for the oxidative addition, following the bisphosphine pathway, however, reveal strong dependence on the Hammett para constants. A reasonable linear correlation $r^{2}=0.954$ has been obtained between $\Delta G$ and $\sigma_{p}$. The correlation diagram is depicted in Figure 5. 


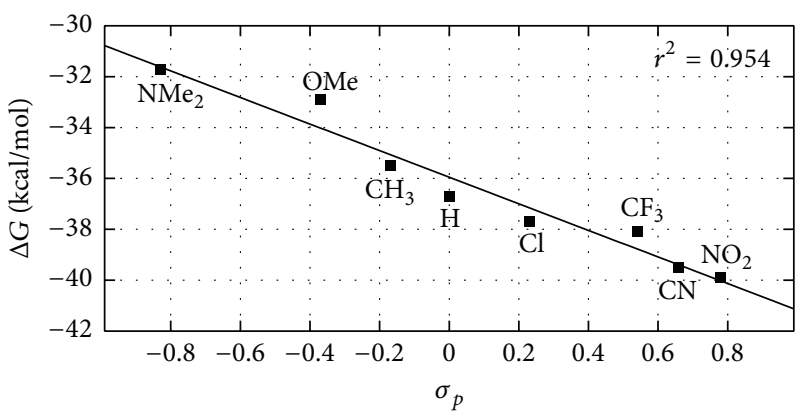

Figure 5: Relationship between the Hammett $\sigma_{p}$ constants and the reaction free energy of the oxidative addition of 4 -substituted iodobenzenes. Correlation equation: $\Delta G=-5.224( \pm 0.464) \cdot \sigma_{p}-$ $35.952( \pm 0.246)$.

The free energy of coordination of iodobenzenes to the highly unsaturated complex 2 shows a peculiar dependence upon the substituent constant (see Table 2). For the electron donating groups, $\mathrm{NMe}_{2}, \mathrm{OMe}$, and $\mathrm{CH}_{3}$, more stable $\eta^{1}$ $\mathrm{C}$ complexes are formed with free energies of only 3.5$5.8 \mathrm{kcal} / \mathrm{mol}$ above that of the sum of 2 and 3 . Neutral and electron withdrawing substituents, however, fall into another category with relative free energies over $10 \mathrm{kcal} / \mathrm{mol}$ with respect to $\mathbf{2}+\mathbf{3}$. Interestingly, the relative stability of transition states 8TS shows an opposite trend revealing the highest barrier $(15.8 \mathrm{kcal} / \mathrm{mol})$ for the methoxy group and the lowest barrier $(11.7 \mathrm{kcal} / \mathrm{mol})$ for the cyano group. On the other hand, the relative free energies of the threecoordinate complexes 9 show no obvious dependence on $\sigma_{p}$. For all substituents the barriers exceed those obtained for the bisphosphine pathway; thus, the monophosphine route (i.e., the dissociative mechanism) is disfavored in all cases.

\section{Conclusion}

A DFT study employing the BP86-D3 theory was carried out utilizing the SMD solvation model with THF as solvent in order to shed some light on the mechanism of the oxidative addition of 4-substituted iodobenzenes on $\mathrm{Pd}(0)-\mathrm{PMe}_{3}$ complexes. The bisphosphine pathway starting from complex $\mathrm{Pd}\left(\mathrm{PMe}_{3}\right)_{2}$ (1) is compared with the monophosphine route, where the addition of iodobenzenes is preceded by the dissociation of the $\mathrm{PMe}_{3}$ ligand resulting in complex 2, as catalyst.

It can be concluded that this elementary step, with $\mathrm{PMe}_{3}$, as ligand, is expected to take place in a very fast reaction. The bisphosphine pathway is predicted to be the viable route regardless of the para substituents for two reasons: (i) the very facile addition of $\mathbf{3} \mathbf{a}-\mathbf{h}$ to $\mathbf{1}$ and (ii) the high dissociation free energy of the $\mathrm{PMe}_{3}$ ligand, which results in an overall barrier of $31.2 \mathrm{kcal} / \mathrm{mol}$ for the monophosphine pathways. The reaction free energy of the oxidative addition shows linear correlation with the Hammett constant of the para substituent, with the most electron withdrawing groups being the most exergonic.

These results suggest that, in accord with previous findings, palladium complexes with basic and sterically not demanding phosphines may serve as highly active catalyst for cross-coupling reactions where oxidative addition is ratedetermining. The strong preference for the bisphosphine pathway, however, emphasizes that the phosphine should be employed at least in a 2:1 ratio with respect to palladium, when the catalyst is formed "in situ."

\section{Conflict of Interests}

The authors declare that there is no conflict of interests regarding the publication of this paper.

\section{Acknowledgments}

The authors thank the Hungarian Scientific Research Fund (K113177) and environmental industry related innovative trans- and interdisciplinary research team development in the University of Pécs knowledge base (SROP-4.2.2.D15/1/KONV-2015-0015) for the financial support. This paper was supported by the János Bolyai Research Scholarship of the Hungarian Academy of Sciences. The authors also thank the Supercomputer Center of the National Information Infrastructure Development (NIIF) Program.

\section{References}

[1] F. Diederich and A. de Mejiere, Eds., Metal Catalyzed CrossCoupling Reactions, Wiley, New York, NY, USA, 2004.

[2] K. C. Nicolaou, P. G. Bulger, and D. Sarlah, "Palladiumcatalyzed cross-coupling reactions in total synthesis," Angewandte Chemie-International Edition, vol. 44, no. 29, pp. 4442-4489, 2005.

[3] K. J. Bonney and F. Schoenebeck, "Experiment and computation: a combined approach to study the reactivity of palladium complexes in oxidation states 0 to IV,' Chemical Society Reviews, vol. 43, no. 18, pp. 6609-6638, 2014.

[4] N. Miyaura and A. Suzuki, "Palladium-catalyzed cross-coupling reactions of organoboron compounds," Chemical Reviews, vol. 95, no. 7, pp. 2457-2483, 1995.

[5] R. Chinchilla and C. Nájera, "Recent advances in Sonogashira reactions," Chemical Society Reviews, vol. 40, no. 10, pp. 50845121, 2011.

[6] J. K. Stille, "The palladium-catalyzed cross-coupling reactions of organotin reagents with organic electrophiles [new synthetic methods (58)]," Angewandte Chemie International Edition, vol. 25 , no. 6 , pp. 508-524, 1986.

[7] C. Cordovilla, C. Bartolomé, J. M. Martínez-Ilarduya, and P. Espinet, "The stille reaction, 38 years later," ACS Catalysis, vol. 5, no. 5, pp. 3040-3053, 2015.

[8] K. Tamao, K. Sumitani, and M. Knmada, "Selective carboncarbon bond formation by cross-coupling of grignard reagents with organic halides. Catalysis by nickel-phosphine complexes," Journal of the American Chemical Society, vol. 94, no. 12, pp. 4374-4376, 1972.

[9] S. Baba and E.-I. Negishi, "A novel stereospecific alkenyl-alkenyl cross-coupling by a palladium- or nickel-catalyzed reaction of alkenylalanes with alkenyl halides," Journal of the American Chemical Society, vol. 98, no. 21, pp. 6729-6731, 1976. 
[10] L. Xue and Z. Lin, "Theoretical aspects of palladium-catalysed carbon-carbon cross-coupling reactions," Chemical Society Reviews, vol. 39, no. 5, pp. 1692-1705, 2010.

[11] J. P. Stambuli, M. Bühl, and J. F. Hartwig, "Synthesis, characterization, and reactivity of monomeric, arylpalladium halide complexes with a hindered phosphine as the only dative ligand," Journal of the American Chemical Society, vol. 124, no. 32, pp. 9346-9347, 2002.

[12] F. Barrios-Landeros and J. F. Hartwig, "Distinct mechanisms for the oxidative addition of chloro-, bromo-, and iodoarenes to a bisphosphine palladium(0) complex with hindered ligands," Journal of the American Chemical Society, vol. 127, no. 19, pp. 6944-6945, 2005.

[13] F. Barrios-Landeros, B. P. Carrow, and J. F. Hartwig, "Effect of ligand steric properties and halide identity on the mechanism for oxidative addition of haloarenes to trialkylphosphine $\operatorname{Pd}(0)$ complexes," Journal of the American Chemical Society, vol. 131, no. 23, pp. 8141-8154, 2009.

[14] A. Kurbangalieva, D. Carmichael, K. K. Hii, A. Jutand, and J. M. Brown, "Oxidative addition to palladium(0) diphosphine complexes: observations of mechanistic complexity with iodobenzene as reactant," Chemistry, vol. 20, no. 4, pp. 1116-1125, 2014.

[15] H. M. Senn and T. Ziegler, "Oxidative addition of aryl halides to palladium(0) complexes: a density-functional study including solvation," Organometallics, vol. 23, no. 12, pp. 2980-2988, 2004.

[16] S. Kozuch, C. Amatore, A. Jutand, and S. Shaik, "What makes for a good catalytic cycle? A theoretical study of the role of an anionic palladium $(0)$ complex in the cross-coupling of an aryl halide with an anionic nucleophile," Organometallics, vol. 24, no. 10, pp. 2319-2330, 2005.

[17] L. J. Goossen, D. Koley, H. L. Hermann, and W. Thiel, "Mechanistic pathways for oxidative addition of aryl halides to palladium(0) complexes: a DFT study," Organometallics, vol. 24, no. 10, pp. 2398-2410, 2005.

[18] M. Ahlquist and P.-O. Norrby, "Oxidative addition of aryl chlorides to monoligated palladium(0): a DFT-SCRF study," Organometallics, vol. 26, no. 3, pp. 550-553, 2007.

[19] K. C. Lam, T. B. Marder, and Z. Lin, "DFT studies on the effect of the nature of the aryl halide $\mathrm{Y}-\mathrm{C}_{6} \mathrm{H}_{4}-\mathrm{X}$ on the mechanism of its oxidative addition to $\mathrm{Pd}^{0} \mathrm{~L}$ versus $\mathrm{Pd}^{0} \mathrm{~L}_{2}$, ' Organometallics, vol. 26, no. 3, pp. 758-760, 2007.

[20] C. L. McMullin, J. Jover, J. N. Harvey, and N. Fey, "Accurate modelling of $\mathrm{Pd}(0)+\mathrm{PhX}$ oxidative addition kinetics," Dalton Transactions, vol. 39, no. 45, pp. 10833-10836, 2010.

[21] F. Proutiere and F. Schoenebeck, "Solvent effect on palladiumcatalyzed cross-coupling reactions and implications on the active catalytic species," Angewandte Chemie-International Edition, vol. 50, no. 35, pp. 8192-8195, 2011.

[22] K. Vikse, T. Naka, J. S. McIndoe, M. Besora, and F. Maseras, "Oxidative additions of aryl halides to palladium proceed through the monoligated complex," ChemCatChem, vol. 5, no. 12, pp. 3604-3609, 2013.

[23] Z. Li, Y. Fu, S.-L. Zhang, Q.-X. Guo, and L. Liu, "Heck-type reactions of imine derivatives: a DFT study," Chemistry-An Asian Journal, vol. 5, no. 6, pp. 1475-1486, 2010.

[24] P. Surawatanawong, Y. Fan, and M. B. Hall, "Density functional study of the complete pathway for the Heck reaction with palladium diphosphines," Journal of Organometallic Chemistry, vol. 693, no. 8-9, pp. 1552-1563, 2008.
[25] A. A. C. Braga, N. H. Morgon, G. Ujaque, and F. Maseras, "Computational characterization of the role of the base in the Suzuki-Miyaura cross-coupling reaction," Journal of the American Chemical Society, vol. 127, no. 25, pp. 9298-9307, 2005.

[26] J. Jover, N. Fey, M. Purdie, G. C. Lloyd-Jones, and J. N. Harvey, "A computational study of phosphine ligand effects in SuzukiMiyaura coupling," Journal of Molecular Catalysis A: Chemical, vol. 324, no. 1-2, pp. 39-47, 2010.

[27] P. Fitton and E. A. Rick, "The addition of aryl halides to tetrakis(triphenylphosphine)palladium(0)," Journal of Organometallic Chemistry, vol. 28, no. 2, pp. 287-291, 1971.

[28] A. D. Becke, "Density-functional exchange-energy approximation with correct asymptotic behavior," Physical Review A, vol. 38, no. 6, pp. 3098-3100, 1988.

[29] J. P. Perdew, "Density-functional approximation for the correlation energy of the inhomogeneous electron gas," Physical Review B, vol. 33, no. 12, pp. 8822-8824, 1986.

[30] M. J. Frisch, G. W. Trucks, H. B. Schlegel et al., Gaussian 09 Revision D.01, Gaussian Inc, Wallingford, Conn, USA, 2009.

[31] S. Grimme, S. Ehrlich, and L. Goerigk, "Effect of the damping function in dispersion corrected density functional theory," Journal of Computational Chemistry, vol. 32, no. 7, pp. 14561465, 2011.

[32] F. Weigend and R. Ahlrichs, "Balanced basis sets of split valence, triple zeta valence and quadruple zeta valence quality for $\mathrm{H}$ to Rn: design and assessment of accuracy," Physical Chemistry Chemical Physics, vol. 7, no. 18, pp. 3297-3305, 2005.

[33] C. Gonzalez and H. B. Schlegel, "An improved algorithm for reaction path following," The Journal of Chemical Physics, vol. 90, no. 4, pp. 2154-2161, 1989.

[34] A. V. Marenich, C. J. Cramer, and D. G. Truhlar, "Universal solvation model based on solute electron density and on a continuum model of the solvent defined by the bulk dielectric constant and atomic surface tensions," The Journal of Physical Chemistry B, vol. 113, no. 18, pp. 6378-6396, 2009.

[35] T. A. Keith, AIMAll (Version 15.05.18), TK Gristmill Software, Overland Park, Kan, USA, 2015, http://aim.tkgristmill.com/.

[36] R. F. W. Bader, Atoms in Molecules-A Quantum Theory, Oxford University Press, Oxford, UK, 1990.

[37] G. Frenking and U. Pidun, "Ab initio studies of transition-metal compounds: the nature of the chemical bond to a transition metal," Journal of the Chemical Society, Dalton Transactions, no. 10, pp. 1653-1662, 1997. 

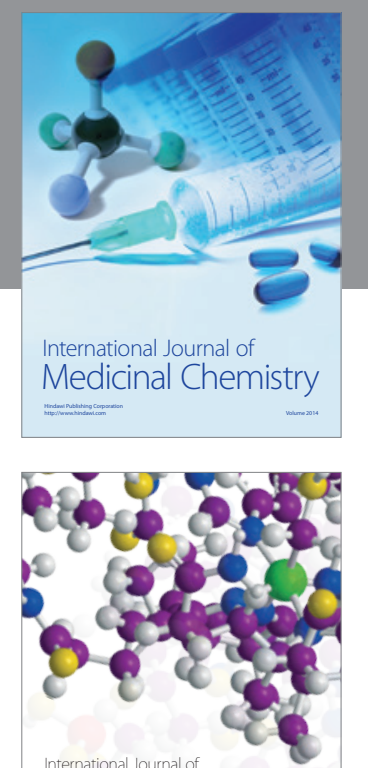

\section{Carbohydrate} Chemistry

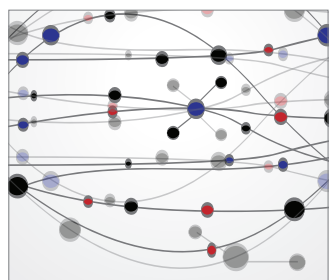

The Scientific World Journal
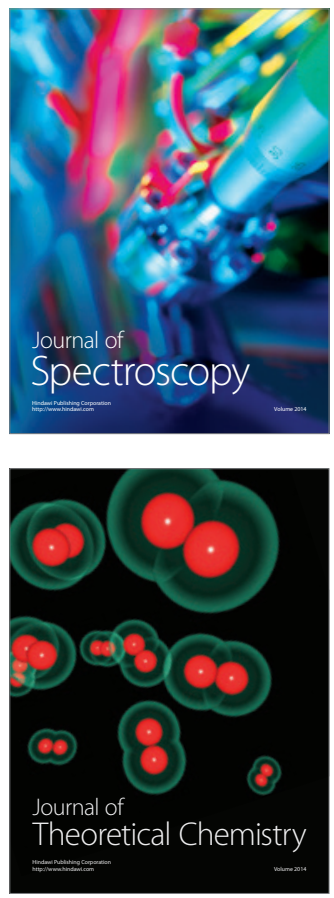
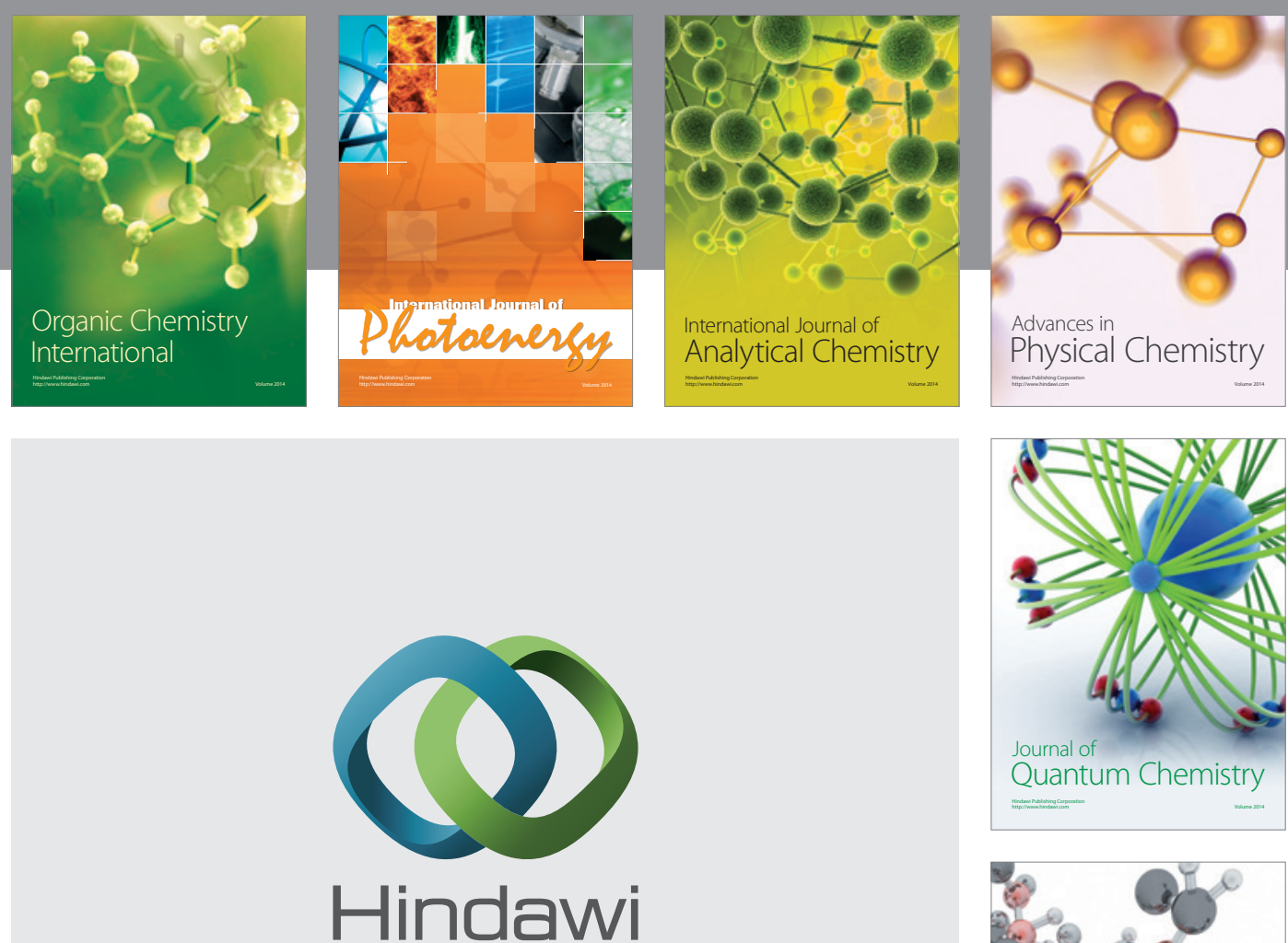

Submit your manuscripts at

http://www.hindawi.com

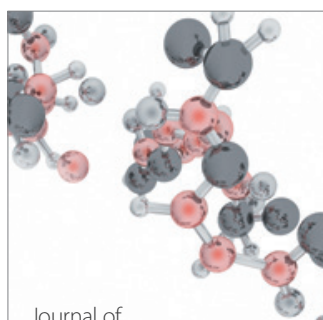

Analytical Methods

in Chemistry

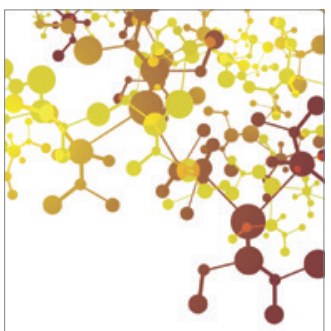

Journal of

Applied Chemistry

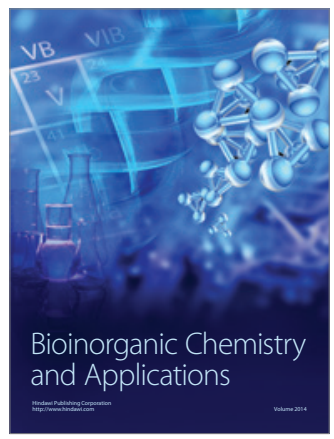

Inorganic Chemistry
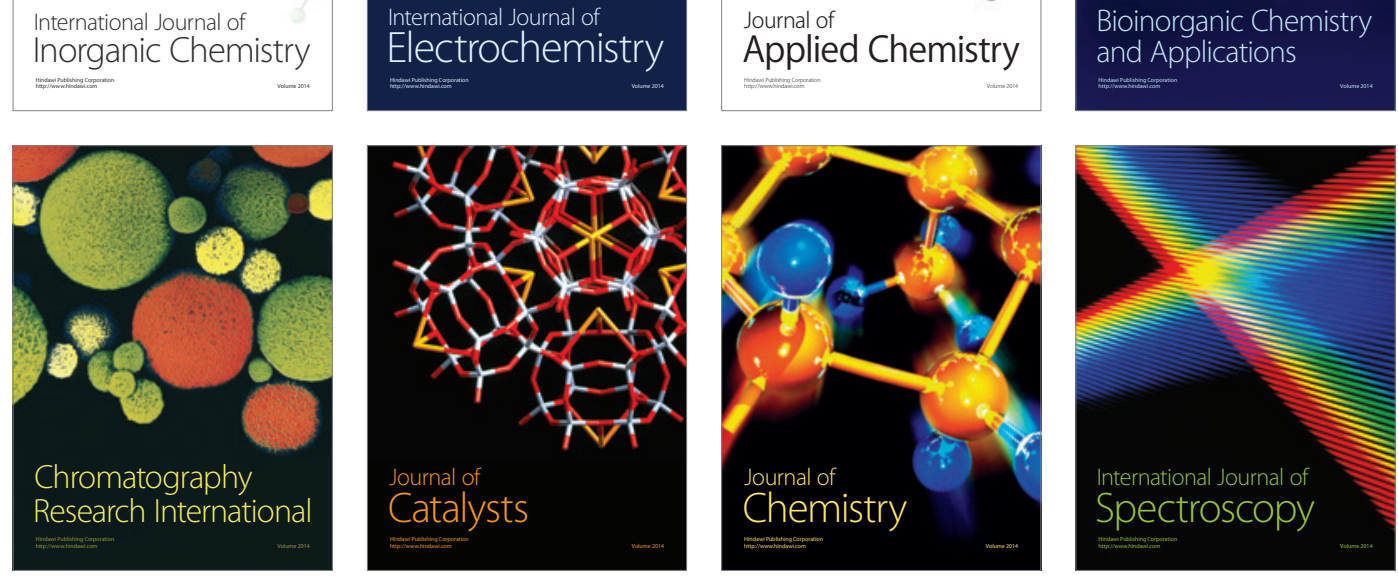